\title{
THE KOBAYASHI INDICATRIX AT THE CENTER OF A CIRCULAR DOMAIN
}

\author{
THEODORE J. BARTH
}

\begin{abstract}
The indicatrix of the Kobayashi infinitesimal metric at the center of a pseudoconvex complete circular domain coincides with this domain. It follows that a nonconvex complete circular domain cannot be biholomorphic to any convex domain. An example shows that a bounded pseudoconvex complete circular domain in $\mathbf{C}^{2}$ need not be taut.
\end{abstract}

Let $V$ be a complex Banach space with norm \|\| . A complete circular domain is a nonempty open set $M \subset V$ such that $\lambda M \subset M$ for all $\lambda \in \mathbf{C}$ with $|\lambda| \leqslant 1$. Hartogs $[5$, p. 76] encountered these domains while expanding analytic functions into series of homogeneous polynomials; Caratheodory [2, p. 104] introduced the term "Kreisgebiet" to describe them. For the purposes of this paper, a semigauge on $V$ is an upper semicontinuous function $p: V \rightarrow[0, \infty)$ such that $p(\lambda v)=|\lambda| p(v)$ for all $\lambda \in \mathbf{C}$ and $v \in V ; p$ is called a gauge if, in addition, \|\|$\leqslant C p$ for some constant $C$.

THEOREM 1. Let $V$ be a complex Banach space. The formulas

$$
M=\{v \in V: p(v)<1\}, \quad p(v)=\inf \{\lambda>0: v \in \lambda M\}
$$

establish a one-to-one correspondence between the complete circular domains $M$ and the semigauges $p$ on $V$. Moreover:

(a) $M$ is bounded if and only if $p$ is a gauge;

(b) $M$ is convex if and only if $p$ is a seminorm;

(c) $M$ is pseudoconvex if and only if $p$ is plurisubharmonic.

In case $V$ has finite dimension:

(d) $M$ is taut [10, p. 199] if and only if $p$ is a continuous plurisubharmonic gauge.

Proof. Standard normed space techniques yield the one-to-one correspondence and properties (a) and (b).

(c) If $p$ is plurisubharmonic, it follows immediately [8, p. 42] that $M$ is pseudoconvex. On the other hand, by definition [8, p. 41], $M$ is pseudoconvex if and only if the function $-\log \delta$ is plurisubharmonic, where $\delta: M \times(V-\{0\}) \rightarrow(0, \infty]$ is defined by

$$
\delta(z, v)=\inf \{|\lambda|: \lambda \in \mathbf{C}, z+\lambda v \notin M\}
$$

Received by the editors October 6, 1982.

1980 Mathematics Subject Classification. Primary 32H15; Secondary 32A07, 32F15.

Key words and phrases. Kobayashi metric, complete circular domain, taut domain, pseudoconvex domain, convex domain. 
Now $p(v)=1 / \delta(0, v)=\exp (-\log \delta(0, v))$ for $v \in V-\{0\}$. Hence, if $M$ is pseudoconvex it follows that $p$ is plurisubharmonic on $V-\{0\}$ and, thus, since $p$ is obviously subharmonic on every complex line through $0, p$ is plurisubharmonic on V.

(d) Recall that $M$ is taut [1,p. 430] if and only if the family of all holomorphic mappings from the unit disk $D=\{w \in \mathbf{C}:|w|<1\}$ into $M$ is normal in the sense that every sequence of such mappings contains a subsequence converging in the compact-open topology or diverging compactly. $\left(\left\{f_{j}\right\}\right.$ diverges compactly if for any compact sets $K \subset D, K^{\prime} \subset M$ there exists an integer $j_{0}$ such that $f_{j}(K) \cap K^{\prime}=\varnothing$ for all $j \geqslant j_{0}$.) If $p$ is a continuous plurisubharmonic gauge, then (a) implies that $M$ is bounded, and it follows (for finite-dimensional $V$ ) that every sequence of holomorphic mappings from $D$ into $M$ contains a subsequence $\left\{f_{j}\right\}$ converging to a holomorphic mapping $f: D \rightarrow V$; since $p \circ f_{j}<1$ and $p$ is continuous, we have $p \circ f \leqslant 1$; by the maximum principle for subharmonic functions, either $p \circ f<1$ (so that $f: D \rightarrow M$ ), or $p \circ f \equiv 1$ (so that $\left\{f_{j}\right\}$ diverges compactly); thus $M$ is taut. On the other hand, if $M$ is taut then (c) implies that $p$ is plurisubharmonic. To complete the proof it will suffice to show that if $M$ is unbounded or $p$ is discontinuous, then $M$ cannot be taut. Now if $M$ is unbounded there are points $v_{j} \in M$ with $\left\|v_{j}\right\| \rightarrow \infty$; the holomorphic mappings $f_{j}: D \rightarrow M$ defined by $f_{j}(w)=w v_{j}$ satisfy $f_{j}(0)=0$ and $\left\|f_{j}(w)\right\| \rightarrow \infty$ for $w \neq 0$, so $M$ is not taut. Finally if $p$ is discontinuous there are points $v_{j} \rightarrow v_{0} \in M$ with $p\left(v_{j}\right) \nrightarrow p\left(v_{0}\right)$; since $p$ is upper semicontinuous we may assume that $p\left(v_{j}\right) \leqslant b<p\left(v_{0}\right)$ for all $j$; the holomorphic mappings $f_{j}: D \rightarrow M$ defined by $f_{j}(w)=w v_{j} / b$ satisfy $f_{j}(0)=0$, while $f_{j}\left(b / p\left(v_{0}\right)\right)=v_{j} / p\left(v_{0}\right) \rightarrow v / p\left(v_{0}\right)$ $\notin M$; thus $M$ is not taut.

Among the more tractable complete circular domains are the complete Reinhardt domains in $\mathbf{C}^{n}$. Recently P. Pflug [9] has shown that every bounded pseudoconvex complete Reinhardt domain is finitely compact with respect to its Carathéodory distance; hence such a domain is taut. Nevertheless, by modifying an example due to N. Kerzman [6, pp. 180-181], we can construct a bounded pseudoconvex complete circular domain in $\mathbf{C}^{2}$ that is not taut. Indeed, by Theorem 1 it suffices to observe that the formula

$$
p(z, w)=\exp \left(\max \left(\log |z|, 1+\sum_{n=1}^{\infty} 2^{-n} \log |n w-z|\right)\right)
$$

defines a plurisubharmonic gauge $p: \mathbf{C}^{2} \rightarrow[0, \infty)$ that is not continuous at the point $z=1, w=0$.

Let $D=\{w \in \mathbf{C}:|w|<1\}$ be the open unit disk, and let $\operatorname{Hol}(X, Y)$ denote the set of holomorphic mappings from $X$ into $Y$. The Carathéodory and Kobayashi differential metrics at the point $z$ of the complex (Banach) manifold $M$ are the semigauges on the complex tangent space $T_{z}(M)$ defined by

$$
E_{M}(v)=\sup \left\{\left|g_{*}(v)\right|: g \in \operatorname{Hol}(M, D) \text { and } g(z)=0\right\}
$$

and

$$
F_{M}(v)=\inf \left\{|u|: u \in T_{0}(D) \text { and } f_{*}(u)=v \text { for some } f \in \operatorname{Hol}(D, M)\right\}
$$


[7,pp. 360-361]. Here we have identified $T_{0}(D)$ with $\mathbf{C}$ so that || denotes the ordinary absolute value. The corresponding complete circular domains

$$
\Gamma_{z}(M)=\left\{v \in T_{z}(M): E_{M}(v)<1\right\} \text { and } \Delta_{z}(M)=\left\{v \in T_{z}(M): F_{M}(v)<1\right\}
$$

are called the indicatrices of these metrics at $z$ [7, p. 399]. In case $M$ is an open subset of the Banach space $V$ and $z=0$, we can identify $T_{z}(M)$ with $V$ [3,pp. 113-114].

THEOREM 2. Let $M$ be a complete circular domain. Then $\Gamma_{0}(M) \supset \Delta_{0}(M) \supset M$.

(a) $\left[2\right.$, Satz 5, p. 120] If $M$ is convex, then $\Gamma_{0}(M)=M$.

(b) If $M$ is pseudoconvex, then $\Delta_{0}(M)=M$.

Proof. The inclusion $\Gamma_{z}(M) \supset \Delta_{z}(M)$ holds at any point $z$ in a complex manifold. Now let $p: V \rightarrow[0, \infty)$ be the semigauge associated with the complete circular domain $M$ by Theorem 1. Let $v \in V$; for each $u>p(v)$ the formula $f(w)=w v / u$ defines a mapping $f \in \operatorname{Hol}(D, M)$ with $f_{*}(u)=v$; thus $F_{M}(v) \leqslant p(v)$. Therefore $\Delta_{0}(M) \supset M$.

(a) [3, Lemma V.1.5, p. 116]. Assume that $M$ is convex. By Theorem $1(\mathrm{~b}), p$ is a continuous seminorm. Let $v \in V$; the Hahn-Banach theorem gives a continuous linear functional $h: V \rightarrow \mathbf{C}$ such that $|h| \leqslant p$ and $h(v)=p(v)$; now $g=\left.h\right|_{M} \in$ $\operatorname{Hol}(M, D), g(0)=0$, and $\left|g_{*}(v)\right|=|h(v)|=p(v)$; thus $E_{M}(v) \geqslant p(v)$.

(b) Assume that $M$ is pseudoconvex. By Theorem 1(c), $p$ is plurisubharmonic. Let $v \in \Delta_{0}(M)$. Then there exist $u \in T_{0}(M)$ and $f \in \operatorname{Hol}(D, M)$ such that $f(0)=0$, $u f^{\prime}(0)=v$, and $F_{M}(v) \leqslant|u|<1$. Expanding $f$ in a power series, we see that the association $w \rightarrow f(w) / w$ extends to a holomorphic mapping $g: D \rightarrow V$ with $g(0)=$ $f^{\prime}(0)$. Take $0<R<1$. For $|w|=R$ we have $p \circ g(w)=p(f(w) / w)=p(f(w)) / R$ $<1 / R$. Since $p \circ g$ is subharmonic, $p \circ g(0)<1 / R$. Letting $R \rightarrow 1$ we get

$$
p(v)=p\left(u f^{\prime}(0)\right)=|u| p\left(f^{\prime}(0)\right)=|u| p \circ g(0) \leqslant|u|<1
$$

i.e., $v \in M$.

COROLlaRY. If the complete circular domain $M$ is biholomorphic to a convex domain, then $M$ is convex.

Proof. A biholomorphic mapping $f: M \rightarrow N$ induces a linear biholomorphic mapping $f_{*}: \Delta_{0}(M) \rightarrow \Delta_{z}(N)$ where $z=f(0)$ [7, p. 399]. If $N$ is convex, then $\Delta_{z}(N)$ is convex [4, Exercise 13, pp. 397-398], hence $\Delta_{0}(M)$ is convex; also $N$ is pseudoconvex and, since pseudoconvexity is biholomorphic invariant, so is $M$; by Theorem $2(\mathrm{~b}), M=\Delta_{0}(M)$ is convex.

\section{REFERENCES}

1. T. J. Barth, Taut and tight complex manifolds, Proc. Amer. Math. Soc. 24 (1970), 429-431.

2. C. Carathéodory, Über die Geometrie der analytischen Abbildungen, die durch analytische Funktionen von zwei Veränderlichen vermittelt werden, Abh. Math. Sem. Univ. Hamburg 6 (1928), 96-145.

3. T. Franzoni and E. Vesentini, Holomorphic maps and invariant distances, North-Holland Math. Studies, 40, Notas Mat., 69, North-Holland, Amsterdam and New York, 1980.

4. L. A. Harris, Schwarz-Pick systems of pseudometrics for domains in normed linear spaces, Advances in Holomorphy, North-Holland Math. Studies, 34, Notas Mat., 65, North-Holland, Amsterdam and New York, 1979, pp. 345-406. 
5. F. Hartogs, Zur Theorie der analytischen Funktionen mehrerer unabhängiger Veränderlichen, insbesondere über die Darstellung derselben durch Reihen, welche nach Potenzen einer Veränderlichen fortschreiten, Math. Ann. 62 (1906), 1-88.

6. N. Kerzman and J. P. Rosay, Fonctions plurisousharmoniques d'exhaustion bornées et domaines taut, Math. Ann. 257 (1981), 171-184.

7. S. Kobayashi, Intrinsic distances, measures and geometric function theory, Bull. Amer. Math. Soc. 82 (1976), 357-416.

8. P. Noverraz, Pseudo-convexité, convexité polynomiale et domaines d'holomorphie en dimension infinie, North-Holland Math. Studies, 3, Notas Mat., 48, North-Holland, Amsterdam, 1973.

9. P. Pflug, About the Carathéodory completeness of all Reinhardt domains, preprint.

10. H. Wu, Normal families of holomorphic mappings, Acta Math. 119 (1967), 193-233.

Department of Mathematics, University of California, Riverside, California 92521 